\title{
Exercício aeróbico intervalado na reabilitação de pacientes com insuficiência cardíaca: revisão sistemática da literatura
}

\author{
Interval aerobic exercise for rehabilitation of patients \\ with heart failure: a systematic review of the literature \\ Tainá Maria de Souza Vidal ${ }^{1}$, Simone Cristina Soares Brandão ${ }^{1}$, Daniella Cunha Brandão², Gilmário Ricarte Batista ${ }^{3}$ \\ ${ }^{1}$ Curso de Pós-graduação em Ciências da Saúde da Universidade Federal de Pernambuco (UFPE) - Recife (PE), Brasil. \\ ${ }^{2}$ Departamento de Fisioterapia da UFPE - Recife (PE), Brasil. \\ ${ }^{3}$ Departamento de Educação Física da Universidade Federal da Paraíba (UFPB) - João Pessoa (PB), Brasil.
}

DOI: http://dx.doi.org/10.7322/abcshs.v38i3.23

\section{RESUMO}

O objetivo deste artigo foi revisar sistematicamente as evidências disponíveis sobre os efeitos na capacidade funcional, atividade simpática cardíaca e qualidade de vida, que constituem os principais indicadores de prognóstico, encontrados em estudos que empregam protocolos de reabilitação com exercício aeróbico intervalado em pacientes com insuficiência cardíaca. Foi realizado um levantamento de dados sobre essa temática a partir das bases de dados MEDLINE, LILACS e SciELO, utilizando cinco descritores em Ciências da Saúde ou Medical Subject Headings, três termos-livres e sete cruzamentos. Na base de dados MEDLINE, via Publisher MEDLINE, cruzando-se as palavras-chave e os termos livres, foram encontrados 155 artigos. Já nas bases de dados LILACS e SciELO, nas quais foi realizada busca nos idiomas inglês, português e espanhol, foram encontrados seis artigos, conjuntamente. Conclui-se, pelas evidências relatadas nesta revisão, que o exercício aeróbico intervalado em pacientes com insuficiência cardíaca (IC) proporciona melhora da capacidade funcional, aferida por meio do $\mathrm{VO}_{2}$ pico, e na qualidade de vida, quando comparado ao exercício aeróbico contínuo, ambos realizados de forma isolada.

Palavras-chave: atividade motora; tolerância ao exercício; saúde.

\begin{abstract}
The purpose of this article was to systematically review the available evidence about the effects on functional capacity, cardiac sympathetic activity and quality of life, which are the main prognostic indicators found in studies employing rehabilitation protocols with interval aerobic exercise in patients with heart failure. Data collection was carried out in the databases MEDLINE, LILACS and SciELO, using five keywords in Health Sciences or Medical Subject Headings, three freeterms and seven combinations. A total of 155 articles were retrieved from the database MEDLINE, via Publisher MEDLINE, by crossing the keywords and free terms. Six articles were found in the databases LILACS and SciELO, in which were made searches in English, Portuguese and Spanish. According to the findings reported herein, it is concluded that interval aerobic exercise provides patients with heart failure with improved functional capacity as measured by $\mathrm{VO}_{2}$ peak, and improved quality of life when compared to continuous aerobic exercise, both performed alone.
\end{abstract}

Keywords: motor activity; exercise tolerance; health. 


\section{INTRODUÇÃO}

A insuficiência cardíaca (IC) caracteriza-se por um grave e crescente problema de saúde pública em todo o mundo com altas taxas de mortalidade e re-hospitalizações ${ }^{1}$, sobretudo em países industrializados com grande população idosa ${ }^{2}$. A IC é uma síndrome complexa, de caráter crônico, resultante de anormalidades funcionais ou estruturais do coração que alteram sua capacidade de enchimento e/ou ejeção ${ }^{3}$. Com o agravamento da IC, o débito cardíaco se torna incapaz de satisfazer a demanda metabólica dos tecidos da periferia ou satisfaz à custa de altas pressões de enchimento ventricular ${ }^{4}$, provocando limitações físico-funcionais, o que, consequentemente, afeta a realização das atividades de vida diária desses indivíduos ${ }^{5}$.

No intuito de amenizar tais implicações, foram criados os programas de Reabilitação Cardíaca (RC), que buscam oferecer adaptações hemodinâmicas, metabólicas, miocárdicas, vasculares, alimentares e psicológicas visando à diminuição da mortalidade cardiovascular e à melhora da qualidade de vida ${ }^{6,7}$. A estratégia de implantação e manutenção do programa de RC deve seguir normas bem estabelecidas de atividade aeróbica e de exercícios resistidos aplicados sobre a musculatura periférica ${ }^{8,9}$.

Apesar de o exercício aeróbico contínuo ainda ser o mais comumente empregado na reabilitação de cardiopatas, há evidências de que o método intervalado permita aos pacientes com IC a realização de séries de exercícios de alta intensidade por períodos relativamente longos, com menor grau de fadiga e maior dispêndio calórico total ${ }^{10,11}$, sendo uma ótima alternativa para facilitar a adaptação da musculatura esquelética para que ossam suportar exercícios de maior intensidade, de forma mais gradativa e mais assimilável, além de quebrar a monotonia do treinamento ${ }^{11}$. Por isso, tem sido observado, na última década, interesse na investigação dos efeitos proporcionados por protocolos de RC que incluem o exercício aeróbico intervalado ${ }^{12,13}$.

Assim, no intuito de propor recomendações para futuras investigações e para a prática clínica atual, o objetivo deste artigo foi revisar sistematicamente as evidências disponíveis sobre os efeitos na capacidade funcional, atividade simpática cardíaca e qualidade de vida, que constituem os principais indicadores de prognóstico, encontrados em estudos que empregaram protocolos de reabilitação com exercício aeróbico intervalado em pacientes com IC.

\section{MÉTODOS}

Trata-se de uma revisão sistemática da literatura que foi realizada a partir das bases de dados Medical Literature Analysis and Retrieval System Online (MEDLINE), Literatura Latino-Americana e do Caribe em Ciências da Saúde (LILACS) e Scientific Electronic Library Online (SciELO), tendo a busca de dados ocorrido de setembro a dezembro de 2012. Foi elaborada uma estratégia para o cruzamento dos Descritores em Ciências da Saúde (DeCS)/Medical Subject Headings (MeSH), palavras-chave para recuperação de assuntos da literatura científica e termos-livres (TL), que são termos não encontrados no DeCS e no MeSH, mas de relevância para a pesquisa.

Na MedLine, foi utilizada a ferramenta de busca PubMed, com a seguinte estratégia de busca utilizando: "Heart failure" (DeCS) AND "Exercise aerobic interval" (TL); "Heart failure" (DeCS) AND "Exercise aerobic interval" AND "Cardiac rehabilitation" (TL) OR "Quality of life" (DeCS) OR "Exercise tolerance" (DeCS) OR "Oxygen consumption" (DeCS) OR "Cardiac sympathetic activity" (TL) OR "Rehabilitation" (DeCS). Na LILACS e na SciELO Regional, foram utilizados os mesmos descritores com seus correspondentes em português e espanhol. Assim, foram contabilizados cinco DeCS, três TL e sete cruzamentos.

Os critérios de inclusão dos estudos encontrados pelas buscas nas bases de dados foram: ser ensaios clínicos randomizados; ter como sujeitos de pesquisa indivíduos com IC submetidos ao exercício aeróbico intervalado realizado somente em esteira ou bicicleta ergométrica; abordar as alterações na capacidade funcional, na atividade simpática cardíaca ou na tolerância ao exercício por meio do consumo de oxigênio ou na qualidade de vida dessa população; e estar publicado nos idiomas português, inglês ou espanhol.

Foram excluídas revisões de literatura, estudos quase-experimentais, estudos de casos, crossover e os que trouxeram outra proposta de intervenção para pacientes com insuficiência cardíaca ou associaram o exercício aeróbico intervalado a outra modalidade terapêutica, seja medicamentosa, cirúrgica ou de exercícios resistidos para musculatura periférica ou respiratória e realizaram avaliação/análise em conjunto. Também foram excluídos trabalhos que abordaram a associação da IC com a tetralogia de Fallot, menopausa, hemodiálise, doença pulmonar obstrutiva crônica e em casos de pré ou pós-transplante cardíaco.

\section{Análise dos dados}

A seleção dos artigos encontrados com a busca nas diferentes bases de dados foi realizada em três etapas. Na primeira etapa, foi realizada a leitura dos títulos dos estudos encontrados. Foram excluídos aqueles que claramente não se enquadravam em qualquer um dos critérios de inclusão deste estudo. Na segunda etapa, foi realizada a leitura dos resumos dos estudos selecionados na primeira etapa e, da mesma forma, foram excluídos aqueles que claramente não se adequaram a qualquer um dos critérios de inclusão preestabelecidos. Na terceira etapa, todos os estudos que não foram excluídos nessas duas primeiras etapas foram lidos na íntegra para seleção dos quais seriam incluídos nesta revisão.

$\mathrm{Na}$ base de dados MEDLINE via PubMed, cruzando-se as palavras-chave e os termos livres, foram encontrados 155 artigos. Já nas bases de dados LILACS e SciELO, nas quais realizou-se busca nos idiomas inglês, português e espanhol, foram encontrados seis artigos, conjuntamente. O fluxo da busca de artigo ao longo da pesquisa, que foi realizada por dois revisores independentes, pode ser verificado no fluxograma apresentado na Figura 1. 


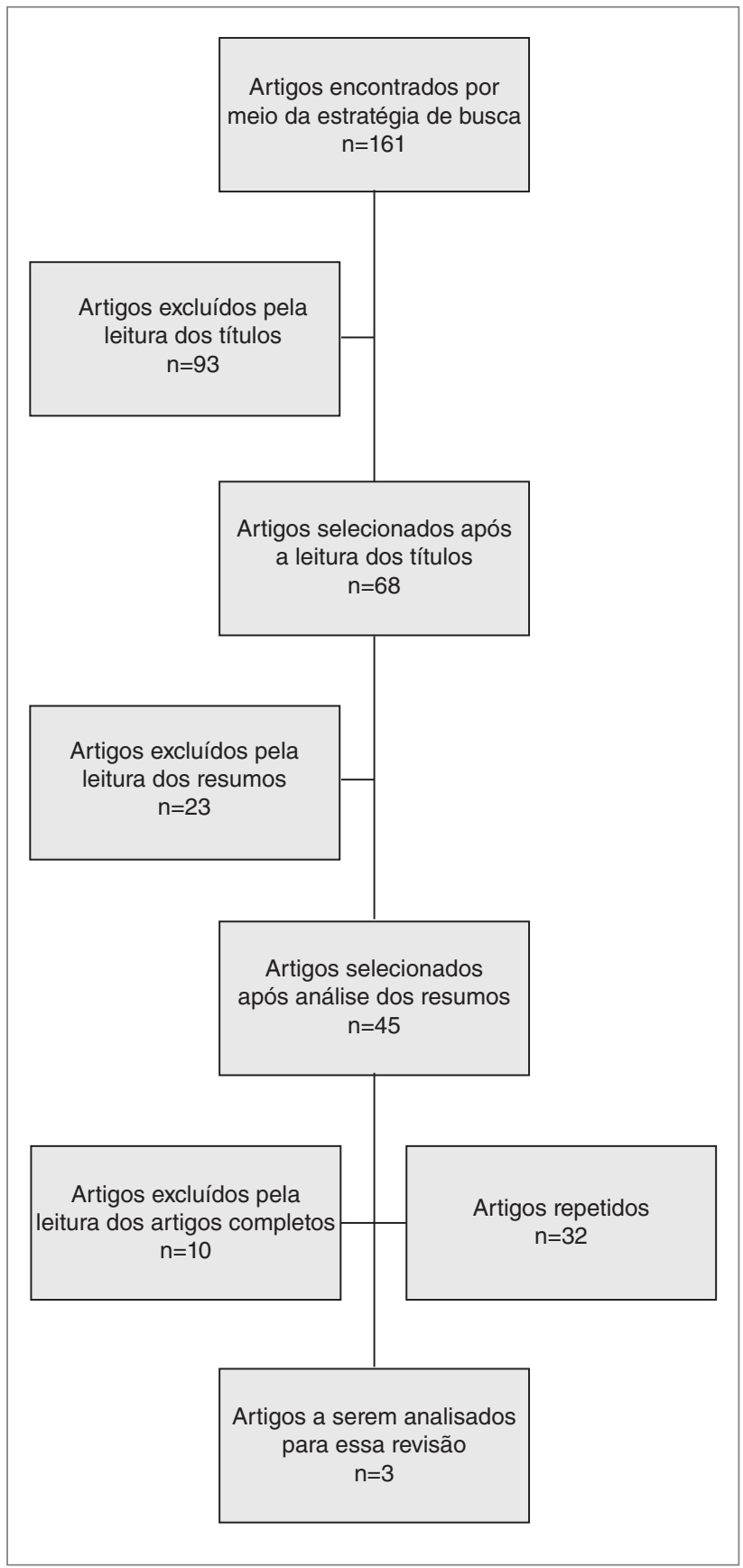

Figura 1: Fluxograma do número de artigos encontrados e selecionados após aplicação dos critérios de inclusão e exclusão segundo descritores e bases de dados

\section{RESULTADOS}

A análise dos artigos que trataram sobre o exercício aeróbico intervalado na IC pode ser observada no Quadro 1 e na Tabela 1.

Um total de três artigos ${ }^{14-16}$ foi selecionado nesta revisão sistemática para analisar a prática do exercício aeróbico intervalado na IC. Observou-se que apenas a partir de 2007 houve interesse na investigação a respeito dos efeitos do exercício aeróbico intervalado em pacientes com IC. Tais estudos se restringiram a algumas populações da Europa e da Ásia, em países como Noruega, Itália e Taiwan.
Quadro 1: Classificação metodológica dos artigos selecionados sobre o exercício aeróbico na insuficiência cardíaca

\begin{tabular}{|c|c|c|c|}
\hline & Wisloff et al. ${ }^{14}$ & Fu et al..$^{15}$ & lellamo et al..$^{16}$ \\
\hline $\begin{array}{l}\text { Critérios de inclusão } \\
\text { especificados }\end{array}$ & Sim & Sim & Sim \\
\hline Grupo controle & Sim & Não & Não \\
\hline Alocação aleatória & Sim & Sim & Sim \\
\hline Sigilo na alocação & Sim & Não & Não \\
\hline Sujeitos "cegos" & Não & Não & Não \\
\hline Terapeutas "cegos" & Não & Não & Não \\
\hline Análise estatística & Sim & Sim & Sim \\
\hline $\begin{array}{l}\text { Comparação estatística } \\
\text { entre grupos }\end{array}$ & Sim & Sim & Sim \\
\hline
\end{tabular}

Houve heterogeneidade dos artigos em relação às médias de idade que variaram entre 62,2 e 75,5 anos e à média de fração de ejeção do ventrículo esquerdo (FEVE), que variou de 29 a 38,3\%. Todos os estudos selecionados compararam o exercício aeróbico intervalado com o exercício aeróbico contínuo, e, em um dos casos, comparou-se ainda com um grupo controle que não realizou qualquer tipo de exercício aeróbico. Além disso, todos os protocolos empregados tiveram duração de 12 semanas com uma frequência semanal que variou entre duas e cinco vezes. A duração das sessões diárias variou de 24 a 38 minutos. Já o pico de intensidade do exercício aeróbico foi de $80 \%$ do $\mathrm{VO}_{2}$, pico até $95 \%$ da frequência cardíaca de pico.

A avaliação da capacidade funcional, em todos os estudos analisados, foi aferida por meio do teste de esforço cardiopulmonar, no qual a principal variável discutida foi o $\mathrm{VO}_{2}$ pico. Somente dois artigos averiguaram o nível de qualidade de vida dos indivíduos com IC submetidos à reabilitação por meio dos seguintes questionários: MacNew, Minnesota Living e SF-36. Apesar do foco da busca realizada, não foi encontrado nenhum estudo que avaliasse a atividade simpática cardíaca.

\section{DISCUSSÃO}

A partir dos anos 2000, as pesquisas com exercício aeróbico intervalado começaram a ser realizadas com atletas em busca de melhores rendimentos durante as competições ${ }^{17}$. Com o passar dos anos, alguns pesquisadores extrapolaram os limites do esporte e vêm investigando o efeito do treino intervalado em variáveis relacionadas às doenças, como na reabilitação cardíaca em pacientes com $\mathrm{IC}^{18,19}$. Essa metodologia possibilita que os períodos de recuperação permitam a realização de curtos períodos de alta intensidade de exercício que não seria conseguido se a prática fosse do tipo contínua (steady-state), promovendo um grande estímulo de exercícios para os músculos periféricos sem produzir um estresse cardiovascular significante ao indivíduo cardiopata ${ }^{20}$.

Em relação à localização das pesquisas, observou-se que os artigos selecionados na presente revisão foram realizados em alguns países da Europa ${ }^{14,16}$ e da Ásia ${ }^{15}$. Porém, essa constatação não está relacionada com a concentração de estudos com interesse na investigação do exercício aeróbico intervalado na IC em 
Tabela 1: Variáveis a serem analisadas dos artigos selecionados sobre o exercício aeróbico intervalado na insuficiência cardíaca de acordo com o autor

\begin{tabular}{|c|c|c|c|}
\hline \multirow{2}{*}{ Variáveis } & \multicolumn{3}{|c|}{ Autor } \\
\hline & Wisloff et al..$^{14}$ & Fu et al. ${ }^{15}$ & lellamo et al. ${ }^{16}$ \\
\hline País & Noruega & Taiwan & Itália \\
\hline Amostra & 27 pacientes (20 homens e 7 mulheres) & 40 pacientes ( 26 homens e 14 mulheres) & 16 pacientes (homens) \\
\hline $\begin{array}{l}\text { Idade média } \\
\text { em anos }\end{array}$ & 75,5 & 67,5 & 62,2 \\
\hline FEVE $^{*}$ & 29 & 38,3 & 31,5 \\
\hline $\begin{array}{l}\text { Intervenções } \\
\text { aplicadas }\end{array}$ & $\begin{array}{l}\text { Treinamento aeróbico contínuo e intervalado } \\
\text { (3x por semana em } 12 \text { semanas) }\end{array}$ & $\begin{array}{l}\text { Treinamento aeróbico contínuo e intervalado } \\
\text { (3x por semana em } 12 \text { semanas) }\end{array}$ & $\begin{array}{l}\text { Treinamento aeróbico contínuo e intervalado } \\
\text { (2 a 5x por semana em } 12 \text { semanas) }\end{array}$ \\
\hline $\begin{array}{l}\text { Variáveis } \\
\text { avaliadas }\end{array}$ & $\begin{array}{l}\text { Consumo de oxigênio de pico }\left(\mathrm{VO}_{2} \text { pico), }\right. \\
\text { FEVE, qualidade de vida }\end{array}$ & $\mathrm{VO}_{2}$ pico, qualidade de vida & $\mathrm{VO}_{2}$ pico, FEVE \\
\hline $\begin{array}{l}\text { Ferramentas } \\
\text { utilizadas }^{\star \star}\end{array}$ & $\begin{array}{c}\text { Teste cardiopulmonar, ecocardiograma, } \\
\text { questionário MacNew }\end{array}$ & $\begin{array}{c}\text { Teste de esforço cardiopulmonar, } \\
\text { questionário SF-36, questionário } \\
\text { Minnesota Living }\end{array}$ & Teste de esforço cardiopulmonar \\
\hline Resultados & $\begin{array}{l}\text { Melhora de todos os aspectos } \\
\text { intra e intergrupos do } \\
\text { exercício aeróbico intervalado }\end{array}$ & $\begin{array}{l}\text { Melhora intragrupo do } \\
\text { exercício aeróbico intervalado }\end{array}$ & $\begin{array}{l}\text { Melhora intra e intergrupos } \\
\text { do exercício aeróbico intervalado }\end{array}$ \\
\hline
\end{tabular}

*Fração de ejeção do ventrículo esquerdo (\%); **Testes, exames e questionários utilizados para análise dos dados

tais continentes. Esse resultado se deve aos critérios de exclusão obedecidos nessa revisão. Existem relatos de diversos estudos realizados na América ${ }^{21}$, inclusive no Brasil ${ }^{22}$, que é um país no qual há grande aplicabilidade clínica do exercício aeróbico intervalado nos protocolos de reabilitação cardíaca em centros de reabilitação. Estes seguem diretrizes atualizadas que indicam a implementação de protocolos que associam o exercício aeróbico, contínuo ou intervalado, com o exercício resistido ${ }^{8,9}$.

Geralmente, os ensaios clínicos randomizados realizados nessa linha de pesquisa incluem poucos pacientes, devido, possivelmente, a melhores condições para controle e operacionalização da pesquisa $^{23,24}$. Os ensaios clínicos randomizados são considerados padrão de excelência para esclarecer a relação causa-efeito. São estudos de alta credibilidade como produtores de evidências científicas, porém podem apresentar dificuldades práticas, como falha na adesão e perda de seguimento, e éticas, em que os benefícios tem que ser superiores aos riscos. Além disso, exigem uma população estável e cooperativa e requerem boa estrutura administrativa, técnica e financeira ${ }^{25}$.

A IC torna-se mais grave com o tempo devido à remodelação ventricular ${ }^{3}$. Assim, com o envelhecimento, percebe-se agravamento dos casos - o que foi observado nos estudos selecionados: apesar da variância de idades, 62 a 75 anos, todos os estudos incluíram indivíduos idosos ${ }^{14,16}$. Esse fato está diretamente relacionado com a deterioração da FEVE, a qual determina insuficiência ventricular quando está abaixo de $40 \%{ }^{4}$, critério de inclusão que foi especificado em todos os artigos selecionados.

Todos os estudos compararam o treinamento aeróbico intervalado com o treinamento aeróbico contínuo ${ }^{14,16}$, que é o tipo de treino tradicionalmente preconizado para pacientes com IC, pois, por questões éticas, não se pode testar uma nova metodologia com a ausência de uma intervenção se já houver alguma assistência que comprovadamente proporcione benefícios aos indivíduos em questão ${ }^{26,27}$.
Comparando-se as duas modalidades de treinamento, apesar de Iellamo et al. ${ }^{16}$ não terem encontrado diferenças na FEVE antes e após o programa de exercícios, Wisloff et al. ${ }^{14}$ apresentaram uma melhora na função sistólica com aumento de 10 pontos percentuais, o que corresponde a 35\% em termos relativos de aumento da FEVE, nos indivíduos que realizaram o exercício aeróbico intervalado de alta intensidade.

Quanto à frequência e à duração do treinamento, foi observado que houve conformidade com as diretrizes de exercício aeróbico que recomendam a realização desse tipo de exercício de três a cinco vezes por semana durante no mínimo 12 semanas $^{9}$, com intensidade variável que pode ser moderada ou alta ${ }^{28,29}$. Nos estudos analisados, as intensidades nas fases de treino do exercício aeróbico intervalado variaram de 75 a $85 \%$ da frequência cardíaca de reserva, $80 \% \mathrm{VO}_{2}$ pico ou 90 a $95 \%$ da frequência cardíaca de pico, ou seja, eram realizados com alta intensidade. Todos com recuperação ativa utilizando baixa ou moderada intensidade ${ }^{14,16}$.

Além das variáveis abordadas pelo objetivo da presente revisão, como valores de $\mathrm{VO}_{2}$ pico e nível de qualidade de vida, os estudos também analisaram resistência à insulina, hemodinâmica cardíaca, biomarcadores inflamatórios, função endotelial, biópsia muscular e análises sanguínea ${ }^{14,16}$. Essas questões não foram discutidas nesta revisão por não se enquadrarem no objetivo traçado. Embora tenha sido um objeto de investigação dessa revisão, não foram encontrados estudos que avaliaram a atividade simpática cardíaca em pacientes com IC que realizaram exercício aeróbico intervalado.

Verificou-se que os instrumentos aplicados são amplamente utilizados nas pesquisas da área de cardiologia esportiva que avaliam a capacidade funcional e a qualidade de vida ${ }^{5,30}$. A avaliação para prescrição do exercício aeróbico intervalado e para quantificação do $\mathrm{VO}_{2}$ pico pré e pós-treinamento nos três estudos revisados foi feita por meio do teste de esforço cardiopulmonar ou ergoespirometria, que é uma metodologia não invasiva que mede 
a capacidade do corpo de realizar as trocas gasosas, fornecendo uma avaliação objetiva da capacidade e/ou limitação ao exercício físico, sendo, assim, considerado padrão ouro para tal fim ${ }^{31}$.

Segundo Dempster e Donnelly ${ }^{32}$, a avaliação da qualidade de vida de uma população com doença cardíaca deve compreender além de um questionamento global sobre os aspectos físicos, psicológicos e sociais, uma avaliação com questionário específico da doença ou do procedimento abordado.

Essa concepção foi aplicada em pelo menos um dos artigos analisados, que utilizou o questionário SF-36 $6^{33}$, composto por 36 itens, agrupados em oito dimensões de saúde: funcionamento do organismo, dor corporal, socialização, saúde mental, vitalidade, percepção geral da saúde, limitações causadas por problemas físicos e limitações por distúrbios emocionais, e tem o propósito de examinar a percepção do estado de saúde pelo próprio paciente; e o questionário específico para a população estudada, Minnesota Living with Heart Failure ${ }^{34}$, que é composto por 21 itens relacionados às causas dos impedimentos decorrentes do problema cardíaco.

Apesar da recomendação, um dos artigos utilizou somente um questionário de qualidade de vida o qual era específico para população cardiopata. O questionário aplicado não foi o Minnesota ${ }^{34}$, que é o mais comumente utilizado ao redor do mundo em pesquisas com pacientes que apresentam IC, foi $\mathrm{MacNew}^{35}$, que contém 27 itens, cada qual graduado em escala de sete itens, desenvolvido para avaliação da qualidade de vida em pacientes após infarto agudo do miocárdio. Após modificações, ele foi validado para população de pacientes com cardiopatia isquêmica, tanto coronarianos estáveis pós-infarto do miocárdio como portadores de disfunção ventricular sintomática. Este instrumento avalia três dimensões de saúde: emocional, física e social. É ainda interessante ressaltar que esse questionário foi validado na língua portuguesa, podendo ser aplicado à população brasileira desde $2005^{36,37}$.

Apesar de não ter sido uma variável avaliada diretamente nos estudos selecionados, o gasto calórico está relacionado com o gasto energético que, por sua vez, pode ser correlacionado com $\mathrm{o}$ aumento de $\mathrm{VO}_{2} \mathrm{pico}^{38}$. Assim, constata-se que treinos mais intensos podem promover adaptações metabólicas de forma mais eficiente e ainda aumentar o metabolismo pós-exercício, o que é importante para o emagrecimento. No caso do exercício aeróbico intervalado, perde-se bem menos tempo para isso ${ }^{39}$. Tal circunstância merece atenção especial por se tratar de indivíduos com IC, devendo-se indicar acompanhamento nutricional, tendo em vista as possíveis deficiências e peculiaridades apresentadas por essa população ${ }^{40}$.

Houve melhora em todas as variáveis avaliadas em todos os estudos selecionados. Um dos artigos descreveu diferença estatisticamente significativa apenas na análise intragrupo ${ }^{15}$. Os outros dois apresentaram superioridade do exercício aeróbico intervalado sobre o contínuo ${ }^{14,16}$. As condições para obtenção desses resultados foram a realização do exercício aeróbico intervalado de alta intensidade em uma frequência de duas a cinco vezes por semana, durante 12 semanas, em pacientes com IC, maiores que 60 anos de idade e com a FEVE menor que $40 \%$.

Conclui-se, pelas evidências relatadas nesta revisão, que o exercício aeróbico intervalado em pacientes com IC proporciona melhora da capacidade funcional, aferida por meio do $\mathrm{VO}_{2}$ pico, e na qualidade de vida quando comparado ao exercício aeróbico contínuo, ambos realizados de forma isolada. Observou-se também melhora dos indicadores de remodelação cardíaca. Assim, foi demonstrada eficácia dessa modalidade de treinamento em idosos com IC.

São necessários mais estudos clínicos randomizados, inclusive em países da América Latina, a fim de comprovar maior eficácia, eficiência e efetividade do treinamento aeróbico intervalado sobre o contínuo em indivíduos com IC. Primariamente, pode-se afirmar que o treino intervalado proporciona maior conforto e estímulo aos pacientes cardiopatas, além de se apresentar como uma intervenção segura.

\section{REFERÊNCIAS}

1. Barretto ACP, Carlo CHD, Cardoso JN, Morgado PC, Munhoz $\mathrm{RT}$, Eid MO, et al. Re-hospitalizações e morte por insuficiência cardíaca. Arq Bras Cardiol. 2008;91(5):335-41.

2. Rossi Neto JM. A dimensão do problema da insuficiência cardíaca do Brasil e do mundo. Rev Soc Cardiol Estado de São Paulo. 2004;14(1):1-10.

3. Bocchi EA, Braga FGM, Ferreira SMA, Rohde LEP, Oliveira WA, Almeida DR, et al. III Diretriz Brasileira de Insuficiência Cardíaca Crônica. Arq Bras Cardiol. 2009;93 Suppl 1:1-71.

4. Rassi S, Barreto ACP, Porto CC, Pereira CR, Calaça BW, Rassi DC. Sobrevida e fatores prognósticos na insuficiência cardíaca sistólica com início recente dos sintomas. Arq Bras Cardiol. 2005;84(4):277-85.

5. Scattolin FAA, Diogo MJD'E, Colombo RCR. Correlação entre instrumentos de qualidade de vida relacionada à saúde e independência funcional em idosos com insuficiência cardíaca. Cad Saúde Pública. 2007;23(11):2705-15.
6. Adams BJ, Carr JG, Ozonoff A, Lauer MS, Balady GJ. Effect of exercise training in supervised cardiac rehabilitation program on prognostic variables from the exercise tolerance test. Am Cardiol. 2008;101(10):1403-7.

7. Barbosa FEJ, Torres JS, Sousa MIF, Lopes EAB, Barbosa JJ. Avaliação da qualidade de vida em pacientes submetidos a um programa de reabilitação cardiovascular semissupervisionado fase II. Saud Pesq. 2011;4(3):363-72.

8. Araújo CGS, Carvalho T, Castro CLB, Costa RV, Moraes RS, Oliveira Filho JA. Normatização dos equipamentos e técnicas da reabilitação cardiovascular supervisionada. Arq Bras Cardiol. 2004;83(5):448-52.

9. Cortez AA, Ferraz A, Nóbrega ACL, Brunetto AF, Herdy AH, Hossr CAC, et al. Diretriz de reabilitação cardiopulmonar e metabólica: aspectos práticos e responsabilidades. Arq Bras Cardiol. 2006;86(1):74-82 
10. Freyssin C, Verkindt C, Prieur F, Benaich P, Maunier S, Blanc P. Cardiac rehabilitation in chronic heart failure: effect of an 8-week, high-intensity interval training versus continuous training. Arch Phys Med Rehabil. 2012;93(8):1359-64.

11. Iellamo F, Manzi V, Caminiti G, Sposato B, Massaro M, Cerrito A, et al. Dose-response relationship of baroreflex sensitivity and heart rate variability to individually-tailored exercise training in patients with heart failure. Int J Cardiol. 2013;166(2):334-9.

12. Winkelmann ER, Chiappa GR, Lima CO, Viecili PR, Stein R, Ribeiro JP. Addition of inspiratory muscle training to aerobic training improves cardiorespiratory responses to exercise in patients with heart failure and inspiratory muscle weakness. Am Heart J. 2009;158(5):1-7.

13. Laoutaris ID, Adamopoulos S, Manginas A, Panagiotakos DB, Kallistratos MS, Doulaptsis C, et al. Benefits of combined aerobic/ resistance/inspiratory training in patients with chronic heart failure. A complete exercise model? A prospective randomised study. Int J Cardiol. 2013;167(5):1967-72.

14. Wisløff U, Støylen A, Loennechen JP, Bruvold M, Rognmo $\varnothing$, Haram PM, et al. Superior cardiovascular effect of aerobic interval training versus moderate continuous training in heart failure patients: a randomized study. Circulation. 2007;(24):3086-94.

15. Fu TC, Wang CH, Lin PS, Hsu CC, Cherng WJ, Huang SC, et al. Aerobic interval training improves oxygen uptake efficiency by enhancing cerebral and muscular hemodynamics in patients with heart failure. Int J Cardiol. 2013;167(1):41-50.

16. Iellamo F, Manzi V, Caminiti G, Vitale C, Castagna C, Massaro $M$, et al. Matched dose interval and continuous exercise training induce similar cardiorespiratory and metabolic adaptations in patients with heart failure. Int J Cardiol. 2013;167(6):2561-5.

17. Billat LV. Interval training for performance: scientific and empirical practice. Special recommendations for middle and longdistance running. Part I: aerobic interval training. Sports Med. 2001;31(1):13-31.

18. Georgiou D, Chen Y, Appadoo S, Belardinelli R, Greene R, Parides MK, et al. Cost-effectiveness analysis of longterm moderate exercise training in chronic heart failure. Am J Cardiol. 2001;87(8):984-8.

19. King J, Broeder C, Browder K, Panton LFACSM. A comparison of interval vs. Steadystate exercise on substrate utilization in overweight women. Med Sci Sports Exerc. 2002;34(5):130-6.

20. Tjønna AE, Stølen TO, Bye A, Volden M, Slørdahl SA, Odegård R, et al. Aerobic interval training reduces cardiovascular risk factors more than a multitreatment approach in overweight adolescents. Clin Sci (Lond). 2009;116(4):317-26.

21. Tasoulis A, Papazachou O, Dimopoulos S, Gerovasili V, Karatzanos $\mathrm{E}$, Kyprianou $\mathrm{T}$, et al. Effects of interval exercise training on respiratory drive in patients with chronic heart failure. Respir Med. 2010;104(10):1557-65.

22. Costa Rosa LFBP, Batista Júnior ML. Efeito do treinamento físico como modulador positivo nas alterações no eixo neuroimunoendócrino em indivíduos com insuficiência cardíaca crônica: possível atuação do fator de necrose tumoral- $\alpha$. Rev Bras Med Esporte. 2005;11(4):238-42.

23. El Dib RP. Como praticar a medicina baseada em evidências. J Vasc Bras. 2007;6(1):1-4.
24. Nobre MRC, Bernardo WM, Jatene FB. A prática clínica baseada em evidências. Parte II - Buscando as evidências em fontes de informação. Rev Assoc Med Bras. 2004;50(1):221-8.

25. Coutinho ESF, Huf G, Bloch KV. Ensaios clínicos pragmáticos: uma opção na construção de evidências em saúde. Cad Saúde Pública. 2003;19(4):1189-93.

26. Milani M, Kozuki RT, Crescêncio JC, Papa V, Santos MDB, Bertini $C Q$, et al. Efeito do treinamento físico aeróbico em coronariopatas submetidos a um programa de reabilitação cardiovascular. Medicina. 2007;40(3):403-11.

27. Corrêa LMA, Santos TSNP, Moura THP, Negrão CE. Alterações Autonômicas na Insuficiência Cardíaca: benefícios do exercício físico. Rev SOCERJ. 2008;21(2):106-111.

28. Nilsson BB, Westheim A, Risberg MA. Long-term effects of a groupbased high-intensity aerobic interval-training program in patients with chronic heart failure. Am J Cardiol. 2008;102(9):1220-4.

29. Nilsson BB, Westheim A, Risberg MA Effects of group-based highintensity aerobic interval training program in patients with chronic heart failure. Am J Cardiol. 2008;102(10):1361-5

30. Abreu DG, Gomes ALM, Mota R, Sirqueira CM, Pessanha R, Madeira R, et al. Reabilitação Cardíaca: Comparação das respostas hemodinâmicas de repouso entre exercícios contra resistivos e exercícios contra resistivos conjugados com exercícios aeróbicos em indivíduos cardiopatas. Rev Trein Desportivo. 2007;8(1):45-52.

31. Yazbek Jr P, Carvalho RT, Sabbag LMS, Battistella LR. Ergoespirometria. Teste de Esforço Cardiopulmonar, Metodologia e Interpretação. Arq Bras Cardiol. 1998;71(5):719-24.

32. Dempster M, Donnelly M. Measuring the health related quality of life of people with ischaemic heart disease. Heart. 2000;83(6):641-44.

33. Ciconelli RM. Tradução para português e validação do questionário genérico de avaliação de qualidade de vida Medical outcomes study 36 Item Short form Health survavey (SF-36). Tese (Doutorado) - Universidade Federal de São Paulo, Escola Paulista de Medicina, São Paulo:1997. p.120.

34. Carvalho VO, Guimarães GV, Carrara D, Bacal F, Bocchi EA. Validação da Versão em Português do Minnesota Living with Heart Failure Questionnaire. Arq Bras Cardiol. 2009;93(1):39-44.

35. Dixon T, Lim LLY, Oldridge NB. The MacNew heart desease healthrelated quality of life instrument: reference data for users. Qual Life Res. 2002;11(2):173-83.

36. Leal A, Paiva C, Höfer S, Amado J, Gomes L, Oldridge N. Evaluative and discriminative properties of the Portuguese MacNew Heart Disease Health-related Quality of Life Questionnaire. Qual Life Res. 2005;14(10):2335-41.

37. Höfer S, Anelli-Monti M, Berger T, Hintringer F, Oldridge N, Benzer W. Psychometric properties of an established heart disease specific health-related quality of life questionnaire for pacemaker patients. Qual Life Res. 2005;14(8):1937-42.

38. Schoenfeld B, Dawes J. High-intensity interval training: applications for general fitness training. J Strength Cond Res. 2009;31(6):44-46.

39. Gosselin LE, Kozlowski KF, DeVinney-Boymel L, Hambridge C. Metabolic response of different high-intensity aerobic interval exercise protocols. J Strength Cond Res. 2012;26(10):2866-71.

40. Sahade V, Montera VSP. Tratamento nutricional em pacientes com insuficiência cardíaca. Rev Nutr. 2009;22(3):399-408. 